\section{POS0151 A PRACTICAL GUIDE FOR THE ASSESSMENT OF PSORIASIS BURDEN IN PATIENTS WITH PSORIATIC ARTHRITIS}

F. R. Kasiem ${ }^{1}$, A. Pasma ${ }^{1}$, I. Tchetverikov ${ }^{2}$, J. Luime ${ }^{1}$, J. Hazes ${ }^{1}$, M. Van Doorn ${ }^{3}$, M. R. Kok ${ }^{4}$, M. Vis ${ }^{1}$ on behalf of CICERO. ${ }^{1}$ Erasmus MC, Rheumatology, Rotterdam, Netherlands; ${ }^{2}$ Albert Schweitzer Hospital, Rheumatology, Dordrecht, Netherlands; ${ }^{3}$ Erasmus MC, Dermatology, Rotterdam, Netherlands; ${ }^{4}$ Maasstad Hospital, Rheumatology \& Clinical Immunology, Rotterdam, Netherlands

Background: Rheumatologists play an important role in the management of patients with Psoriatic Arthritis (PsA). With PsA being a multifaceted disease, it can be challenging to assess the impact of psoriatic skin symptoms, next to the musculoskeletal complaints. We have previously shown that a dermatology-specific Health-related Quality of Life (HRQoL) questionnaire provides insight into the burden of skin symptoms, in contrast to a general HRQoL questionnaire. ${ }^{1}$ When treating PsA patients, specific questions on skin symptoms are necessary in order to assess the burden of psoriasis.

Objectives: To create a set of questions that can easily identify PsA patients with a high psoriasis burden in daily rheumatology clinical practice.

Methods: Data from patients receiving usual care were used from the Dutch south west Psoriatic Arthritis (DEPAR) cohort, consisting of newly diagnosed PsA patients included between July 2013 and March 2020. The two dermatology-specific HRQoL questionnaires used were the Skindex-17 and Dermatology Life Quality Index (DLQI), both with a one-week recall period.

First, an exploratory principal component analysis (PCA) with varimax rotation was performed on both questionnaires combined, to identify underlying latent traits. Subsequently, items were dichotomized on their median frequencies. And a 2-parameter logistic (2PL) model was fitted for each latent trait. Item characteristic curves were plotted for each latent trait. Item selection took place based on the discrimination and difficulty of the items. Per latent trait, we selected 2 to 3 items distributed far apart across the latent trait. A flowchart was made with the selected questions.

Results: In total, 413 patients with completed Skindex-17 and DLQI questionnaires at baseline were included. Mean (sd) age was 50.3 (13.4) years and median (IQR) Psoriasis Area and Severity Index (PASI) score 2 (0.5-4.2). The PCA gave the best fit with two underlying clusters of questions, namely psychosocial $(n=20)$ questions and questions regarding physical symptoms $(n=7)$. Three questions were selected to assess psychosocial impact and two for impact of physical symptoms (Figure 1). Questions should be asked in chronological order. Cluster 1: Psychosocial

The first question is if the patient was embarrassed by their skin condition. If answered with "never", the patient experienced the lowest burden. When answered with "rarely/sometimes" or "often/always", continue to the second question. Ask the patient if they tended to stay at home because of their skin condition. If answered with "never", they experienced a higher burden. If answered with "rarely/sometimes" or "often/always", continue to the last question. Ask the patient if their skin condition has prevented them from working or studying. If not, the

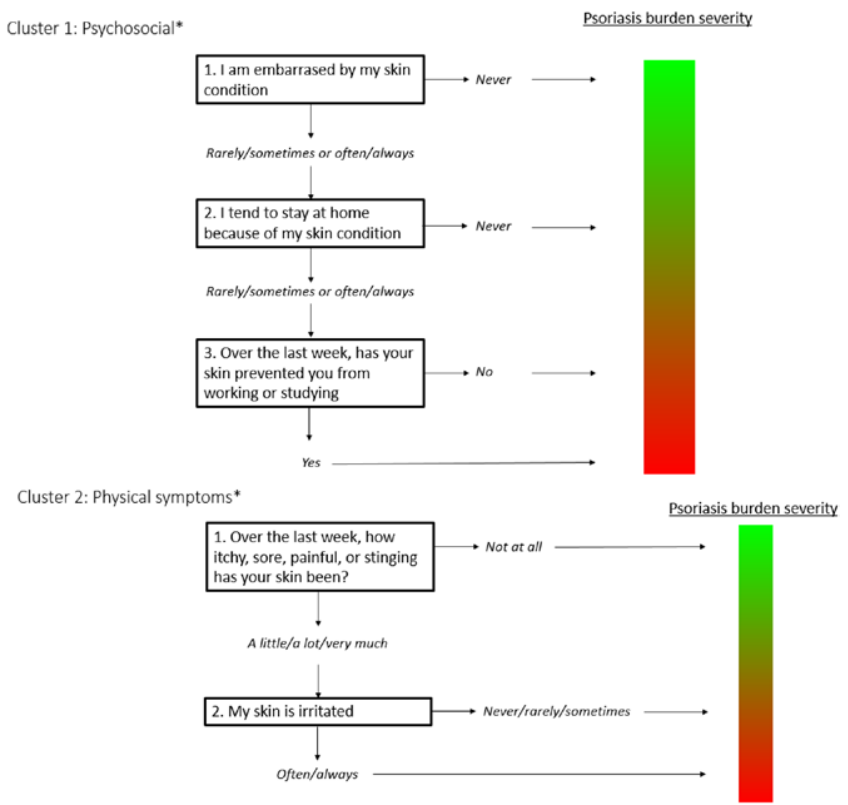

- Questions are to be asked in chronological order

Figure 1. Flowchart of questions assessing psoriasis burden. patient experienced a higher burden and if answered with "yes", they experienced the highest burden in this domain

Cluster 2: Physical symptoms

First ask the patient how itchy, sore, painful or stinging their skin was. If they answer with "not at all", they experienced the lowest burden. If answered with "a little/a lot/very much", continue to the second question. Ask if their skin was irritated. If answered with "never/rarely/sometimes", they experienced a higher burden. If answered with "often/always", the patient experienced the highest burden in this domain.

Conclusion: We have created a practical guide for rheumatologists to distinguish PsA patients with a high psoriasis burden from those with a lower burden. With a minimum of two and a maximum of five questions, both psychosocia burden of psoriasis and burden of physical symptoms can be easily identified in daily clinical practice.

\section{REFERENCES:}

[1] Kasiem FR, Kok MR, Tchetverikov I, Wervers K, Hazes J, Luime J, et al. AB0786 Impact of psoriasis severity on Health-Related Quality of Life in early psoriatic arthritis: results from real world data, the DEPAR study. Annals of the Rheumatic Diseases. 2020;79:1691-2.

Disclosure of Interests: None declared

DOI: 10.1136/annrheumdis-2021-eular.2477

\section{HPR - Adapting and individualising care}

\section{POS0152-HPR REMOTE CLINICAL MANAGEMENT: INCORPORATING ELECTRONIC ASSESSMENT OF PATIENTS WITH RHEUMATIC DISEASES INTO STANDARD CLINICAL PATHWAYS DURING THE COVID-19 PANDEMIC: A PILOT STUDY}

T. Malley ${ }^{1}$, J. Jackman ${ }^{1}$, S. Manderson ${ }^{1}$, L. Saldana Pena ${ }^{1}$, E. Evans ${ }^{1}$, J. Barrett ${ }^{2}$, A. Soni ${ }^{2}$, R. Luqmani ${ }^{2} .{ }^{1}$ Nuffield Orthopaedic Centre, Rheumatology, Oxford, United Kingdom; ${ }^{2}$ The Nuffield Department of Orthopaedics, Rheumatology and Musculoskeletal Sciences, Rheumatology, Oxford, United Kingdom

Background: Many patients with rheumatic disease require immunosuppressive medication putting them at high risk of COVID-19 infection. Reduced staffing in rheumatology due to redeployment to COVID-19 work, limited out patient capacity and patient vulnerability have had a major impact on our ability to review our patients to assess their condition and treatment (by face-to-face, video or telephone consultations). Novel strategies are essential to safely and effectively treat patients with rheumatic disease whilst minimising their risk of exposure to COVID-19 infection. Objectives: The objective was to develop a digital solution to help deliver safe, efficient and effective care for patients with rheumatic diseases. The aim was to produce a system that allowed us to integrate data recorded directly by patients with information held in our electronic health records to provide a virtual review of care.

Methods: An online questionnaire was used to collect clinical information, including validated disease activity measures, to conduct a remote assessment in 175 patients awaiting follow-up appointments. This assessment was integrated within our electronic health records (EHR). The questionnaire contained meas ures of disease activity (DAS28 or BASDAI); patient reported outcomes; patient preferences regarding the urgency and type of appointment; any recent problems or changes in medication. This information was imported into a database for clinician review, together with previous clinical records and results of relevant investigations, to inform clinical decisions and to decide on the safest and most appropriate timing for follow-up. Report letters were sent to the patient and their primary care providers.

Results: Of the 175 patients (149 with RA and 26 with AS), 108 patients (89/149 [60\%] with RA [mean age $=64$; female $=65 \%$ ] and $19 / 26$ [73\%] with AS [mean age $=45$; female $=54 \%$ ]) submitted responses over a 6 -week period based on which clinical decisions were made. The mean questionnaire completion time was 19 minutes for RA responders and 16 minutes for AS responders. Non responders $(67 / 175$ [mean age $=61$; female $=63 \%$ ]) remained on our list of patients awaiting follow-up arrangements to be made. Sixty-nine responders (64\%) had stable disease therefore did not require any changes to their treatment and were offered an appointment within the next 6 months, of whom $12(11 \%)$ requested face-to-face follow-up. Of the remaining 39 - with less stable disease - requiring more rapid follow-up assessment, 22 patients (56\%) required a face-to-face consultation to consider treatment change. So far 9 of these patients have had follow-up, of whom 6 necessitated treatment escalation (Methotrexate increase $n=2$; anti-inflammatory increase $n=2$; intramuscular steroid $n=1$; anti-TNF escalation $n=1$ ) Thirty-nine patients $(36 \%)$ provided feedback on the process of completing the questionnaire, $85 \%$ of whom used a mobile phone and the remainder used a computer or tablet. The majority $(70 \%)$ found it "extremely easy" or "somewhat 